\title{
Erratum to: Phenolic alkaloids from Menispermum dauricum inhibits apoptosis of cerebral ischemia in rats
}

\author{
Qi Guo ${ }^{1} \cdot$ Hui $\mathrm{Su}^{2} \cdot \mathrm{Qi} \mathrm{Jiang}^{3} \cdot$ Xianghui $\mathrm{Qi}^{1} \cdot$ Yunming $\mathrm{Su}^{2} \cdot$ Zhiguo Wang $^{4}$
}

Published online: 22 February 2016

(C) Springer-Verlag Wien 2016

Erratum to: Monatsh Chem (2015) 146:511-520

DOI 10.1007/s00706-014-1327-1

The original version of this article unfortunately contained a mistake.

The corresponding author of this paper is Z. Wang, not Q. Guo.

The online version of the original article can be found under doi:10.1007/s00706-014-1327-1.

Zhiguo Wang

guoqi608@ujs.edu.cn

1 School of Medical Science and Laboratory Medicine,

University of Jiangsu, Zhenjiang, China

2 Heilongjiang University of Chinese Medicine, Haerbin, China

3 Beijing Chaoyang Second Hospital, Beijing, China

4 Experimental Research Center, Academy of Chinese Medical Sciences, Beijing, China 